\title{
Constant Resistance During Proportional Speed Provoked Higher Lower Limb Proximal Musculature Recruitment than Distal Musculature in Young Healthy Adults
}

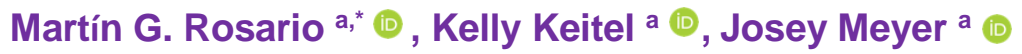 \\ a Physical Therapy Program, Texas Woman's University, Dallas Campus, Texas \\ *Corresponding author Email: mrosario1@twu.edu ; Telephone: 241-689-6500 \\ DOI: https://doi.org/10.34256/ijpefs21310 \\ Received: 28-08-2021, Revised: 14-09-2021; Accepted: 18-09-2021; Published: 24-09-2021
}

\begin{abstract}
The lack of exercise in society today often leads to severe muscle loss and poor physical performance. Training methods targeting specific weakened muscle groups can help prevent or counteract muscle loss. This study aimed to analyze how the lower extremity muscles are activated when pushing a sled with constant resistance at two different speeds. Twenty-six participants with an average age of 23.77 years consented to having electromyography surface electrodes placed along the gluteus maximus (GMax), gluteus medius (GMed), tibialis anterior (TA), and gastrocnemius (GA) of their dominant leg. Muscle activation levels were then measured while the participant walked and ran with and without sled resistance. The study results showed that muscle activation was comparable during all trials and was not influenced by speed or constant resistance. However, the muscle activation for GMax and GMed was significantly higher than the activation levels exhibited by GA and TA. While pushing a sled has been shown to impact all studied musculature similarly, adding resistance to the movement can affect gait parameters such as stride length and cadence. Our findings support the use of sled training in patients with hip pathologies who are seeking to strengthen their GMax and GMed.
\end{abstract}

Keywords: Sled, Contant Resistant, Hip Musculature, Resistant Exercise

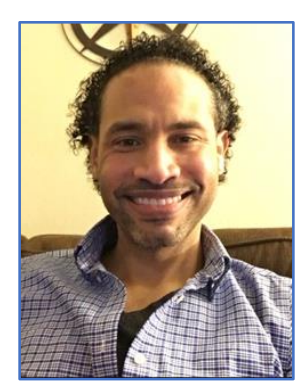

\section{Martin G. Rosario PT, Ph.D.,} CSFI, ATRIC. Obtained his Ph.D. in Neuroanatomy from the University of Puerto Rico Medical Sciences Campus. Master in Physical Therapy from the University of Puerto Rico Medical Sciences Campus School of Physical Therapy. Bachelor in Biology with a Major in Human Focus from University of Puerto Rico Bayamon Campus. Currently serves as an Assistant Professor at Texas Woman's University (TWU) School of Physical Therapy Dallas, Clinical Researcher at an HIV community clinic La Perla de Gran Precio in Puerto Rico, and Director/Coordinator of Motor Control Research Laboratory at TWU Dalla.

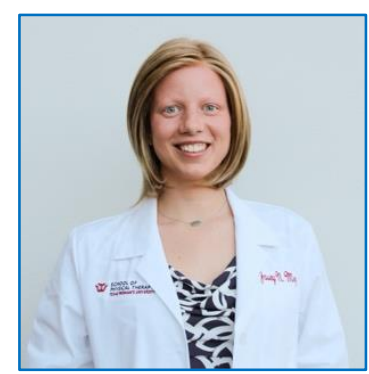

Josey Meyer, SPT. Currently enrolled in the DPT program at Texas Woman's University (TWU). Bachelor of Arts in Biology with a minor in Interdisciplinary Studies from Hardin-Simmons University.

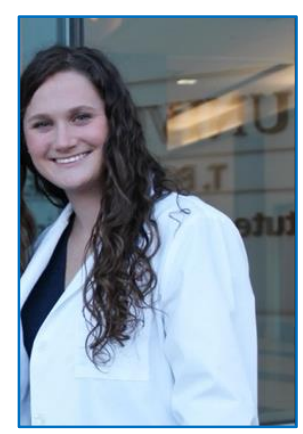

Kelly Keitel, SPT. Currently enrolled in the DPT program at Texas Woman's University (TWU). Bachelor of Science in Exercise Science from Illinois State University 


\section{Introduction}

As a natural part of our aging process, we start to lose muscle mass throughout the body in the development of what is referred to as sarcopenia. In 2016, Giallauria et al., [1] stated that as we get older, the skeletal muscle on the human body progressively reduces and provokes a parallel reduction in muscle strength. Reduced mobility and physical inactivity are some of the reasons prompting sarcopenia; nevertheless, movements and weight-bearing activities could halt or at the very least slow down muscle mass loss [2]. Typically, the body's musculature reduces in size and ability to function adequately as we get older; therefore, the main population affected by sarcopenia is the aged community, especially those who are physically inactive [1]. Sarcopenia is estimated to be prevalent in more than $50 \%$ of adults over the age of eighty years old [3]. However, alarmingly, even young children are experiencing a decrease in muscular fitness in developed countries around the globe as a result of "exercise deficit disorder," a term that describes the severe lack of physical activity in younger generations [4].

Resistance training (RT) is one of the most influential and proven tools to prevent sarcopenia and muscle atrophy is resistance training (RT) [1]. RT is the key factor in developing fundamental movement skills, such as running, jumping, and quickly changing directions, as this type of training involves progressively increasing various loads and movement velocities [3]. This training technique can use elastic bands, plyometrics, free weights, and training machines [5]. The evidence established that RT benefits could come from just two sessions per week, lasting fifteen to twenty-minutes each [6]. The RT that can be used includes controlled movements for each major muscle group and does not require the use of very heavy resistance [6]. The benefits of RT include significant improvements in muscle torque and a reduction in overall injury rates in the lower extremity in youth [6]. Research has shown that RT is also extremely beneficial for older adults in helping to improve bone health, manage cardiovascular diseases, and slow down muscular breakdown [7].

With RT, the objective is to improve the patient's muscular strength and endurance without fatiguing the muscle [3]. A sled is an RT tool used to improve gait or running speed while assessing the subject's ability to push a sled against resistance [8]. Some of the distinct advantages of using a sled for RT have been mentioned in the literature; for instance, the study by Kawamori et al., [8] stated the superior benefits on musculature when towing a sled weighing $30 \%$ of participants' body mass as compared to unresisted sprinting, proving that as more resistance is added, the amount of force required by the participant increases.

The current study will use a sled that provides constant resistance to allow individuals to perform lowresistance, high-repetition training without pushing them to fatigue. The XPO Trainer sled was employed to provide constant resistance over various speeds. Previous studies by Rosario et al., [9] investigated the impact of the XPO trainer on lower limb musculature and showed that faster neuromuscular recruitment occurs in the gastrocnemius (GA) and tibialis anterior (TA) muscles as speed increases. Pushing the resistance sled while walking and running causes a reduction in muscle activation time due to faster neuromuscular recruitment; therefore, using this form of training allows for the isolation of the GA and TA in order to improve motor control and motor coordination [9]. Previous studies have also found that muscle activation occurred later or was delayed in walking while pushing the sled in comparison to tasks involving solely walking. This also advocates that there is an added advantage of pushing the sled versus walking when referring to maximal activation of the quadriceps muscle [9]. The results showed that using the XPO trainer can have a positive effect on neuromuscular recruitment speed and peak muscle activation [9].

This study emphasizes the impact of RT on the neuromuscular activation of the gluteus medius (GMed) and gluteus maximus (GMax) based on the importance of these muscles for proper gait and balance [10]. Nunes [10] tested the activation of the GMed and gluteus minimus while walking and running in asymptomatic, physically active individuals and found that the GMed was at its peak activation during the stance phase of gait. The timing of this peak activation emphasizes the critical role of the GMed in the stabilization of the pelvis, which is vital in weightbearing activities to have proper body mechanics [10]. The GMed was also found to have delayed and shorter activation if the individual also experienced patellofemoral pain; this delay in activation of the GMed caused greater hip adduction, while the delay in GMax caused greater hip internal rotation while running [11].

In contemplation of all the information above, the intention of this proposed study is to assess the 
activation of proximal muscles, such as the gluteus medius and gluteus maximus, while walking-pushing and running-pushing the sled. Because the XPO sled will provide the same level of resistance while the participant is walking or running, this factor will allow the fatigue and muscle activation levels between the two tasks to be more accurately compared. Therefore, the objective of this study was to evaluate the influence of using the XPO trainer sled at slow and fast speeds on the hip (GMed and GMax) and leg (GA and TA) muscle activity and time for activation in young, apparently healthy adults.

\section{Methods}

This study recruited 5 males and 21 females with an average age of 23.77 years +/- 3.09 years. Participants were screened for eligibility based on the inclusion and exclusion criteria. Eligible participants signed an informed consent form before participating.

Following consent and cardiovascular assessment, electromyography (EMG) surface electrodes were placed. Participants were shaved using a razor, when applicable, in the areas where the EMG surface electrodes were to be placed. The participants then had the EMG surface electrodes placed by one of the co-principal investigators on the following muscles: gluteus maximus, gluteus medius, tibialis anterior, and gastrocnemius of the participant's dominant leg.

Gait Protocol: The XPO trainer is the sled used in this study for the push protocols. The XPO trainer is a sled that weighs $60-$ lbs and measures $43^{\prime \prime} \mathrm{H} \times 43.5^{\prime \prime} \mathrm{L}$ $x 35^{\prime \prime} \mathrm{W}$. Participants were first instructed to push the XPO trainer over a distance of $40 \mathrm{ft}$ while walking, followed by sprinting as a "warm up. "Once this warmup was completed, each participant went through four protocols: 1) walk, 2) walk push, 3) run, and 3) run push protocol. For all tasks, EMG data collection was started by the investigator at the point where the participants were told to "start." Once they reached the $40 \mathrm{ft}$ mark, participants were told to "stop" and walk back to the starting position while the XPO trainer was brought back to the start for them. Participants repeated each task twice.

In the walk (W) and run protocol (R), participants walked at a self-selected pace or sprinted as fast as they could over a $40 \mathrm{ft}$ distance. Usually, the $\mathrm{W}$ protocol is followed by the walking push protocol (WP). R tasks was followed by the run push protocol $(R P)$. In these protocols ( $W$ and $R$ ), the participant did not use the XPO trainer.
In the WP, participants walked a $40 \mathrm{ft}$ distance while pushing the XPO trainer. In the RP, participants sprinted a distance of $40 \mathrm{ft}$ while pushing the XPO trainer. Subjects were instructed to push the sled as hard and as fast as possible.

\subsection{Data Analysis}

The EMG data were then placed through a filter and rectified to normalize the maximal voluntary contraction (MVC) data to the muscle activations within the tasks for each participant. The data were processed and downloaded into spreadsheets. The time for each gait task (in seconds) was normalized to the percentage of the task $(0 \%-100 \%)$. Subsequently, the investigators identified the highest activation points for each muscle during all trials with the corresponding percentage of time in the gait cycle. The average of each data point was estimated, and the mean maximal activation and timing of the corresponding task were calculated.

The means were then placed into the SPSS Data Analysis 25 system for repeated-measures ANOVA. In this study, a $P$ value of 0.05 , was considered significant.

\section{Results}

Table 1 presents the demographic characteristics of the participants. There were five men and 21 women who participated in this study. The average age was $23.77 /-3.09$ with an average weight (lbs) of $139.9+/-20.71$. Of the 26 participants, two wore corrective lenses, while the other 24 did not.

Tables $2 a$ and $2 b$ show the comparisons of EMG amplitudes for all muscles in the walk and WP tasks. A significant difference was identified with a decrease in time for the GMax min AMP in the WP when compared to walking. Another significant difference was that the maximum time for the GA was longer for WP than during walking. GMax had a lower min AMP with WP, and GA had a higher percentage of time activated when pushing the sled.

Tables $3 \mathrm{a}$ and $3 \mathrm{~b}$ illustrate the comparisons of EMG amplitudes for all muscles among the RP tasks. The GA had a significantly shorter activation time when running while pushing the sled compared to running without the sled $(p=0.05)$. The amplitudes of the other muscles were comparable between the groups. 
Table 1 Demographic data of all participants

\begin{tabular}{|c|c|}
\hline Characteristics & Participant Data \\
\hline Age & $23.77+/-3.09$ \\
\hline Gender & $\begin{array}{c}\text { Male }=5 \\
\text { Female }=21\end{array}$ \\
\hline Height (in) & $66.5+/-3.92$ \\
\hline Weight (lb) & $139.9+/-20.71$ \\
\hline BMI (kg/m^2) & $21.95+/-3.21$ \\
\hline Heart Rate (bpm) & $77.38+/-15.08$ \\
\hline Systolic BP (mmHg) & $118.82+/-15.75$ \\
\hline Diastolic BP (mmHg) & $76.27+/-9.09$ \\
\hline Sat O2 (\%) & $99+/-.01$ \\
\hline Leg Dominance & $\begin{array}{c}\mathrm{R}=23 \\
\mathrm{~L}=3\end{array}$ \\
\hline Glasses & $\begin{array}{c}\text { Glasses }=2 \\
\text { No glasses }=24\end{array}$ \\
\hline
\end{tabular}

Table 2a Comparisons of EMG AMPLITUDE for GMAX and GMED among tasks. Results of repeated measure ANOVA performed comparing single and dual. Significance level set at $\mathrm{p} \leq 0.05$.

\begin{tabular}{|c|c|c|c|}
\hline GMAX & $\begin{array}{l}\text { Walk } \\
\text { Means and SD }\end{array}$ & $\begin{array}{l}\text { Walk Push } \\
\text { Means and SD }\end{array}$ & P-value \\
\hline MAX \% TIME & $45.76+/-19.31$ & $56.26+/-21.25$ & 0.80 \\
\hline MAX AMP & $70.24+/-24.08$ & $209.26+/-588.82$ & 0.61 \\
\hline MIN \% TIME & $29.55+/-33.31$ & $40.11+/-20.95$ & 0.22 \\
\hline MIN AMP & $50.94+/-16.75$ & $32.29+/-21.81$ & 0.001 \\
\hline AVG AMP & $63.11+/-21.13$ & $70.44+/-45.86$ & 0.09 \\
\hline GMED & Means and SD & Means and SD & P-Value \\
\hline MAX \% TIME & $41.89+/-25.58$ & $47.55+/-21.31$ & 0.38 \\
\hline MAX AMP & $66.99+/-51.06$ & $161.13+/-289.6$ & 0.84 \\
\hline MIN \% TIME & $34.48+/-34.56$ & $22.04+/-28.16$ & 0.11 \\
\hline MIN AMP & $33.97+/-20.01$ & $34.82+/-21.42$ & 0.89 \\
\hline AVG AMP & $44.66+/-27.94$ & $55.29+/-44.27$ & 0.93 \\
\hline $\begin{array}{l}\wedge \mathrm{GMAX}=\text { Glute } \\
\wedge \mathrm{GMED}=\text { Glute } \\
\wedge \mathrm{P}=\mathrm{P}-\text { Value } \\
\wedge \mathrm{S} . \mathrm{D} .=\text { Standa } \\
\text { ^P-Value }>.05 \\
\text { *P-Value }<.05 \\
\wedge \text { AMP = Amplit } \\
\text { ^MIN=minim }\end{array}$ & $\begin{array}{l}\text { uscles } \\
\text { cles }\end{array}$ & & \\
\hline
\end{tabular}


Table 2b Comparisons of EMG AMPLITUDE for TA and GA among tasks. Results of repeated measure ANOVA performed comparing single and dual. Significance level set at $p \leq 0.05$.

\begin{tabular}{|l|l|l|l|}
\hline Tibialis Anterior & $\begin{array}{l}\text { Walk } \\
\text { Means and SD }\end{array}$ & $\begin{array}{l}\text { Walk Push } \\
\text { Means and SD }\end{array}$ & P-value \\
\hline MAX \% TIME & $46.41+/-21.26$ & $40.6+/-17.42$ & 0.28 \\
\hline MAX AMP & $100.03+/-250.3$ & $61.33+/-25.97$ & 0.45 \\
\hline MIN \% TIME & $22.7+/-28.21$ & $20.27+/-27.05$ & 0.72 \\
\hline MIN AMP & $6.3+/-2.77$ & $6.2+/-2.37$ & 0.91 \\
\hline AVG AMP & $25.52+/-43.14$ & $19.25+/-6.89$ & 0.52 \\
\hline Gastrocnemius & Means and SD & Means and SD & P-Value \\
\hline MAX \% TIME & $30.29+/-16.47$ & $53.7+/-17.24$ & 0.001 \\
\hline $\begin{array}{l}\text { MAX AMP } \\
\text { MIN \% TIME }\end{array}$ & $26.76+/-63.45$ & $101.05+/-26.53$ & 0.46 \\
\hline $\begin{array}{l}\text { MIN AMP } \\
\text { AVG AMP }\end{array}$ & $5.81+/-4.76$ & $4.88+/-2.1$ & 0.32 \\
\hline $\begin{array}{l}\text { P=P-Value } \\
\text { ^S.D. =Standard Deviation } \\
\text { *P-Value>.05 is not significant } \\
\text { ^AMP=Amplitude } \\
\text { ^MIN=minimum, MAX=Maximal }\end{array}$ & $18.48+/-9.6$ & $22.23+/-6.41$ & 0.54 \\
\hline
\end{tabular}

Table 3a Comparisons of EMG AMPLITUDE for GMAX and GMED among tasks. Results of repeated measure ANOVA performed comparing single and dual. Significance level set at $p \leq 0.05$.

\begin{tabular}{|l|l|l|l|}
\hline GMax & $\begin{array}{l}\text { Run } \\
\text { Means and SD }\end{array}$ & $\begin{array}{l}\text { Run Push } \\
\text { Means and SD }\end{array}$ & P-value \\
\hline MAX \% TIME & $63.51+/-20.55$ & $57.19+/-15.65$ & 0.28 \\
\hline MAX AMP & $\begin{array}{l}204.47+/- \\
173.21\end{array}$ & $\begin{array}{l}534.73+/- \\
1667.65\end{array}$ & 0.22 \\
\hline MIN \% TIME & $14.81+/-33.31$ & $21.13+/-33.6$ & 0.46 \\
\hline MIN AMP & $50.46+/-15.76$ & $52.62+/-16.36$ & 0.84 \\
\hline AVG AMP & $81.94+/-29.8$ & $150.74+/-327.47$ & 0.69 \\
\hline GMed & Means and SD & Means and SD & P-Value \\
\hline MAX \% TIME & $62.04+/-22.18$ & $60.28+/-16.76$ & 0.79 \\
\hline
\end{tabular}




\begin{tabular}{|l|l|l|l|}
\hline MAX AMP & $\begin{array}{l}952.47+/- \\
2283.84\end{array}$ & $\begin{array}{l}843.59+/- \\
2079.33\end{array}$ & 0.81 \\
\hline MIN \% TIME & $13.16+/-21.81$ & $7.98+/-17.52$ & 0.52 \\
\hline MIN AMP & $33.68+/-19.64$ & $37.14+/-20.73$ & 0.58 \\
\hline AVG AMP & $262.91+/-$ & $219.26+/-441.88$ & 0.71 \\
\hline $\begin{array}{l}\text { ^GMAX=Gluteus Maximus Muscles } \\
\text { ^GMED=Gluteus Medius Muscles }\end{array}$ & & \\
^P=P-Value \\
^S.D.=Standard Deviation \\
^P-Value>.05 is not significant \\
*P-Value<.05 is significant \\
^AMP=Amplitude \\
^MIN=minimum, MAX=Maximal
\end{tabular}

Table 3b Comparisons of EMG AMPLITUDE for TA and GA among tasks. Results of repeated measure ANOVA performed comparing single and dual. Significance level set at $\mathrm{p} \leq 0.05$.

\begin{tabular}{|l|l|l|l|}
\hline Tibialis Anterior & $\begin{array}{l}\text { Run } \\
\text { Means and SD }\end{array}$ & $\begin{array}{l}\text { Run Push } \\
\text { Means and SD }\end{array}$ & P-value \\
\hline MAX \% TIME & $61.52+/-17.48$ & $59.83+/-16.09$ & 0.75 \\
\hline MAX AMP & $152.25+/-215.17$ & $102.67+/-98.17$ & 0.33 \\
\hline MIN \% TIME & $9.81+/-19.18$ & $6.14+/-14.22$ & 0.59 \\
\hline MIN AMP & $7.67+/-3.42$ & $6.89+/-3.29$ & 0.38 \\
\hline AVG AMP & $48.89+/-45.31$ & $34.98+/-16.84$ & 0.15 \\
\hline Gastrocnemius & Means and SD & Means and SD & P-Value \\
\hline MAX \% TIME & $77.07+/-28.04$ & $63.24+/-14.3$ & 0.05 \\
\hline MAX AMP & $199.58+/-150.45$ & $150.32+/-62.1$ & 0.07 \\
\hline $\begin{array}{l}\text { MIN \% TIME } \\
\text { MIN AMP }\end{array}$ & $15.8+/-18.12$ & $20.9+/-30.59$ & 0.45 \\
\hline $\begin{array}{l}\text { AVG AMP } \\
\text { AP=P-Value } \\
\text { ^S.D. =Standard Deviation } \\
\text { ^P-Value>.05 is not significant } \\
\text { *P-Value<.05 is significant } \\
\text { ^AMP=Amplitude } \\
\text { ^MIN=minimum, MAX=Maximal }\end{array}$ & $5.28+/-2.7$ & $5.78+/-2.34$ & 0.60 \\
\hline
\end{tabular}


Table 4 Comparison of Timing of Maximal Muscle Activation Between Muscles During Walk Push. Results of repeated measure ANOVA performed comparing muscles. Significance level set at $\mathrm{p} \leq 0.05$.

\begin{tabular}{|c|c|c|c|}
\hline MAX \% TIME & Means and SD & Means and SD & P-Value \\
\hline & GMAX: 56.26 +/- 21.51 & $\begin{array}{l}\text { GMED: } 47.55+/-21.31 \\
\text { GA: } 53.7+/-17.24 \\
\text { TA: } 63.24+/-14.3\end{array}$ & $\begin{array}{l}0.13 \\
0.65 \\
0.22\end{array}$ \\
\hline & GMED: 47.55 +/- 21.31 & $\begin{array}{l}\text { GA: } 53.7+/-17.24 \\
\text { TA: } 63.24+/-14.3\end{array}$ & $\begin{array}{l}0.27 \\
0.05\end{array}$ \\
\hline & GA: $53.7+/-17.24$ & TA: $63.24+/-14.3$ & 0.09 \\
\hline \multirow[t]{4}{*}{ MAX AMP } & Means and SD & Means and SD & P-Value \\
\hline & GMAX: $209.5+/-588.82$ & $\begin{array}{l}\text { GMED: } 161.13+/-289.6 \\
\text { GA: } 101.05+/-26.53 \\
\text { TA: } 150.32+/-62.1\end{array}$ & $\begin{array}{l}0.62 \\
0.27 \\
0.54\end{array}$ \\
\hline & GMED: $161.13+/-289.6$ & $\begin{array}{l}\text { GA: } 101.05+/-26.53 \\
\text { TA: } 150.32+/-62.1\end{array}$ & $\begin{array}{l}0.59 \\
0.91\end{array}$ \\
\hline & GA: $101.05+/-26.53$ & TA: $150.32+/-62.1$ & 0.60 \\
\hline \multirow[t]{4}{*}{ MIN \% TIME } & Means and SD & Means and SD & P-Value \\
\hline & GMAX: $40.11+/-20.95$ & $\begin{array}{l}\text { GMED: } 22.04+/-28.16 \\
\text { GA: } 26.56+/-23.29 \\
\text { TA: } 20.9+/-30.59\end{array}$ & $\begin{array}{l}0.05 \\
0.89 \\
0.05\end{array}$ \\
\hline & GMED: $22.04+/-28.16$ & $\begin{array}{l}\text { GA: } 26.56+/-23.29 \\
\text { TA: } 20.9+/-30.59\end{array}$ & $\begin{array}{l}0.59 \\
0.88\end{array}$ \\
\hline & GA: $26.56+/-23.29$ & TA: $20.9+/-30.59$ & 0.47 \\
\hline \multirow[t]{4}{*}{ MIN AMP } & Means and SD & Means and SD & P-Value \\
\hline & GMAX: $32.29+/-21.81$ & $\begin{array}{l}\text { GMED: } 34.82+/-21.42 \\
\text { GA: } 4.88+/-2.1 \\
\text { TA: } 5.78+/-2.34\end{array}$ & $\begin{array}{l}0.59 \\
0.001 \\
0.001\end{array}$ \\
\hline & GMED: $34.82+/-21.42$ & $\begin{array}{l}\text { GA: } 4.88+/-2.1 \\
\text { TA: } 5.78+/-2.34\end{array}$ & $\begin{array}{l}0.001 \\
0.001\end{array}$ \\
\hline & GA: $4.88+/-2.1$ & TA: $5.78+/-2.34$ & 0.45 \\
\hline \multirow[t]{4}{*}{ AVG AMP } & Means and SD & Means and SD & P-Value \\
\hline & GMAX: $70.44+/-45.86$ & $\begin{array}{l}\text { GMED: } 55.29+/-44.27 \\
\text { GA: } 22.23+/-6.41 \\
\text { TA: } 44.12+/-14.93\end{array}$ & $\begin{array}{l}0.13 \\
0.01 \\
0.01\end{array}$ \\
\hline & GMED: $55.29+/-44.27$ & $\begin{array}{l}\text { GA: } 22.23+/-6.41 \\
\text { TA: } 44.12+/-14.93\end{array}$ & $\begin{array}{l}0.01 \\
0.25\end{array}$ \\
\hline & GA: $22.23+/-6.41$ & TA: $44.12+/-14.93$ & 0.05 \\
\hline
\end{tabular}


$\wedge \mathrm{TA}=$ Tibialis Anterior Muscle

$\wedge \mathrm{GA}=$ Gastrocnemius Muscle

$\wedge \mathrm{GMAX}=$ Gluteus Maximus Muscles

$\wedge$ GMED $=$ Gluteus Medius Muscles

$\wedge P=P-$ Value

$\wedge$ S.D. $=$ Standard Deviation

$\wedge \mathrm{P}$-Value $>.05$ is not significant

$*$ P-Value $<.05$ is significant

S.D. $=$ Standard Deviation

Table 5 Comparison of Timing of Maximal Muscle Activation Between Muscles During Run Push. Results of repeated measure ANOVA performed comparing muscles. Significance level set at $\mathrm{p} \leq 0.05$.

\begin{tabular}{|c|c|c|c|}
\hline MAX \% TIME & Means and SD & Means and SD & P-Value \\
\hline & GMAX: $57.19+/-15.65$ & $\begin{array}{l}\text { GMED: } 60.28+/-16.76 \\
\text { GA: } 63.24+/-14.3 \\
\text { TA: } 59.83+/-16.09\end{array}$ & $\begin{array}{l}0.52 \\
0.30 \\
0.58\end{array}$ \\
\hline & GMED: $60.28+/-16.76$ & $\begin{array}{l}\text { GA: } 63.24+/-14.3 \\
\text { TA: } 59.83+/-16.09\end{array}$ & $\begin{array}{l}0.53 \\
0.93\end{array}$ \\
\hline & GA: $63.24+/-14.3$ & TA: $59.83+/-16.09$ & 0.47 \\
\hline \multirow[t]{4}{*}{ MAX AMP } & Means and SD & Means and SD & P-Value \\
\hline & GMAX: $534.73+/-1667.65$ & $\begin{array}{l}\text { GMED: } 843.59+/-2079.33 \\
\text { GA: } 150.32+/-62.1 \\
\text { TA: } 102.67+/-98.17\end{array}$ & $\begin{array}{l}0.44 \\
0.33 \\
0.28\end{array}$ \\
\hline & GMED: 843.59 +/- 2079.33 & $\begin{array}{l}\text { GA: } 150.32+/-62.1 \\
\text { TA: } 102.67+/-98.17\end{array}$ & $\begin{array}{l}0.08 \\
0.06\end{array}$ \\
\hline & GA: $150.32+/-62.1$ & TA: $102.67+/-98.17$ & 0.90 \\
\hline \multirow[t]{4}{*}{ MIN \% TIME } & Means and SD & Means and SD & P-Value \\
\hline & GMAX: $21.13+/-33.6$ & $\begin{array}{l}\text { GMED: } 7.98+/-17.51 \\
\text { GA: } 20.9+/-30.59 \\
\text { TA: } 6.14+/-14.22\end{array}$ & $\begin{array}{l}0.09 \\
0.98 \\
0.05\end{array}$ \\
\hline & GMED: 7.98 +/- 17.51 & $\begin{array}{l}\text { GA: } 20.9+/-30.59 \\
\text { TA: } 6.14+/-14.22\end{array}$ & $\begin{array}{l}0.09 \\
0.81\end{array}$ \\
\hline & GA: $20.9+/-30.59$ & TA: $6.14+/-14.22$ & 0.05 \\
\hline \multirow[t]{4}{*}{ MIN AMP } & Means and SD & Means and SD & P-Value \\
\hline & GMAX: $52.62+/-16.36$ & $\begin{array}{l}\text { GMED: } 37.14+/-20.73 \\
\text { GA: } 5.78+/-2.34 \\
\text { TA: } 6.89+/-3.29\end{array}$ & $\begin{array}{l}0.001 \\
0.001 \\
0.001\end{array}$ \\
\hline & GMED: $37.14+/-20.73$ & $\begin{array}{l}\text { GA: } 5.78+/-2.34 \\
\text { TA: } 6.89+/-3.29\end{array}$ & $\begin{array}{l}0.001 \\
0.001\end{array}$ \\
\hline & GA: $5.78+/-2.34$ & TA: $6.89+/-3.29$ & 0.77 \\
\hline AVG AMP & Means and SD & Means and SD & P-Value \\
\hline
\end{tabular}




\begin{tabular}{|l|l|l|l|} 
& GMAX: $150.74+/-327.47$ & GMED: $219.26+/-441.88$ & 0.41 \\
& & GA: 44.12+/- 14.93 & 0.19 \\
& TA: $34.98+/-16.84$ & 0.16 \\
\cline { 2 - 4 } & GMED: $219.26+/-441.88$ & GA: $44.12+/-14.93$ & 0.05 \\
& TA: $34.98+/-16.84$ & 0.05 \\
\cline { 2 - 4 } & GA: 44.12 +/- 14.93 & TA: $34.98+/-16.84$ & 0.91 \\
\hline ^TA=Tibialis Anterior Muscle & \\
^GA=Gastrocnemius Muscle & & \\
^GMAX=Gluteus Maximus Muscles & \\
^GMED=Gluteus Medius Muscles & & \\
^P=P-Value & & \\
^S.D.=Standard Deviation & & \\
^P-Value $>$.05 is not significant & & \\
*P-Value<.05 is significant & & \\
S.D. $=$ Standard Deviation &
\end{tabular}

Table 4 shows the comparison of the timing of maximal muscle activation between muscles during walking push. The minimum muscle activation was significantly higher while walking and pushing a sled in both the GMax and GMed when compared to GA and TA $(p=0.001)$. When comparing the average muscle activation for each muscle group, the GMax muscle had significantly greater muscle activation than both the GA and TA groups $(p=0.01)$. The GMed muscle also had a significantly greater average muscle activation than the GA $(p=0.01)$.

Table 5 compares the timing of maximal muscle activation between the muscles during RP. The minimum muscle activation was significantly higher while running and pushing a sled in both the GMax and GMed when compared to GA and TA $(p=0.001)$. The GMax minimum muscle activation was also significantly higher than that of the GMed ( $p=0.001)$.

\section{Discussion}

The intention of this study was to evaluate the influence of using the Sled at low and fast speeds on hip and leg muscle activity in young, apparently healthy adults. The study presented two main findings, the first being that muscle activation is similar to $\mathrm{W}$ and WP as well as R and RP, and the second finding is that the GMax and GMed exhibited higher activation than the GA and TA when pushing the sled.

As stated above, the first outcome of this study illustrated that amplitudes of W and WP, as well as $\mathrm{R}$ and $\mathrm{RP}$, are comparable. One explanation for these results is based on the assumption that while the amplitude of the muscles remained relatively constant, other gait parameters were influenced when the speed and resistance of the tasks were adjusted. Previous studies using the XPO Trainer Sled have shown that when compared to similar non-pushing activities, pushing the sled causes a decrease in stride length and velocity, along with decreases in the cadence of the participants' gait [12]. By decreasing cadence, the participants spent a greater amount of time on double limb support, which helped to improve balance and stability [12]. Based on these results, we infer that these similarities in muscle activation can be used to achieve a symmetrical gait pattern and increase motor control of gait-related movements. Future studies could compare gait parameters and muscle activation between pushing and pulling tasks.

Another potential explanation for the amplitude of the lower extremity muscles being similar throughout the tasks is that the core or trunk muscles are more engaged than the lower extremity muscles when speed and resistance are adjusted. A study analyzing the differences in postural adjustments while pushing an object showed that activation of both trunk muscles and lower extremity musculature significantly increased while pushing [13]. Trunk musculature plays a significant role in stabilizing the spine and controlling posture in tasks that involve pushing an object [13]. However, while the trunk muscles stabilize the spine, with poor pelvic control over medio-lateral directions, the ability of the spine to control in the frontal plane is reduced [14]. As stated by Powers [14], pelvic control plays a vital role in dynamic trunk stability; therefore, lower extremity muscles such as the GMax and GMed are integral in providing trunk stability [14] and are also important players when considering pushing 
activities such as the sled. Our study furthers the notion that the lower extremity is not the primary musculature in response to changes in gait speed and resistance level. The current study neglected to collect maximal muscle contraction from the trunk musculature, making this a limitation. A future study that compared GMax, GMed, GA, and TA muscle activation to that of the erector spinae of trunk musculature during $\mathrm{W}, \mathrm{WP}, \mathrm{R}$, and RP tasks could potentially reveal whether the trunk musculature exhibits an increase in activation when increasing gait speed with constant resistance.

The second discovery of this study found that GMax and GMed exhibited higher activation than GA and TA when pushing the resistance sled. Likewise, among both pushing tasks, the GA and TA exhibited similar activation patterns, suggesting that pushing a resistance sled causes the hip muscles to be more engaged than the leg muscles. From this, we can infer that while utilizing such an RT sled, the hip musculature has both pushing and stability roles. As Nunes et al., [10] discussed, strong hip musculature, specifically the GMax and GMed, are necessary to stabilize the pelvis and hip while controlling lower limb movements, such as running and other weight-bearing activities. A previous study by Powers [14] showed that the hip muscles that maintain a level pelvis in the frontal plane play an important role in stabilization, which is important because if GMax and GMed do not activate higher than the GA and TA, it would be difficult to elicit forward progression during gait. Neto, et. al., [15] level for the glute muscles, with the GMax being one of the primary hip extensors, and hip extension is a significant kinematic mover for both step-ups and pushing activities.

Based on the Neto study and our results, it can be inferred that sled pushing should be recommended for individuals who require GMax and GMed strengthening such as hip weakness or hip pathology. In a previous study by Rosario (2020), it was found that if the XPO Trainer Sled is used as an RT mechanism, it will assist in gait kinematics as well as provide neuromuscular benefits to the patients who chose this mode of resistance training [9]. While future studies should focus on the impact of hip and leg pathologies, they should also look at the muscular timing of the GMax and GMed muscles when pushing a resistance sled in comparison to performing other activities to discern if there is a difference between the activation times.

\section{Conclusion}

The purpose of this study was to evaluate the effect of using the XPO Trainer Sled at low and fast speeds on hip and leg muscle activity in young, seemingly healthy adults. The most significant finding was that the hip-proximal musculature was more strongly activated when pushing the sled than the lower extremity muscles. This information can help decide which specific group of individuals will most benefit from resistance sled training. This study also deepens the understanding of the impact of pushing an adaptable resistant sled on the hip musculature. Future research should look at the time of onset for which each muscle is activated, how long it is activated for, and if there are any delays or compensations within each muscle. Future studies should also take time to explore the neuromuscular effects of pushing this particular sled on postural muscles, such as those of the trunk, to discern how these muscles are activated in healthy individuals. Including a wide range of participants who are healthy versus those with hip and leg pathologies would be beneficial to include in a future study to comprehensively understand the differences among targeted populations. One limitation of variability that occurs within this study is the inability to push all participants against the same speed, as subjects utilize a self-selected speed and, therefore, individualized levels of resistance. Because the resistance of the sled is velocity dependent, the participants pushed against varying levels of resistance when they ran at different speeds. Future studies should explore the impact of this sled at a dictated speed at a set pace to understand muscle activation under specified conditions. As a final remark, our results indicate that the GMax, GMed, TA, and GA are all active when using the resistance sled; therefore, each can benefit from sled training. Hence, in light of our findings, we recommend that clinicians use the XPO Trainer Sled as a mode of RT to help train individuals with lower extremity pathologies.

\section{References}

[1] F. Giallauria, A. Cittadini, N.A. Smart, \& C. Vigorito, Resistance training and sarcopenia, Monaldi Archives for Chest Disease, 84(1-2) (2016) 738. [DOI] [PubMed]

[2] C.E. Kasper, A.L. McNulty, A.J. Otto, \& D.P. Thomas, Alterations in skeletal muscle related to impaired physical mobility: an empirical model, Research in Nursing \& Health, 16(4) (1993) 265-273. [DOI] [PubMed] 
[3] Maren S Fragala, Eduardo L Cadore, Sandor Dorgo, Mikel Izquierdo, William J Kraemer, Mark D Peterson, Eric D Ryan, Resistance Training for Older Adults: Position Statement from the National Strength and Conditioning Association. Journal of Strength \& Conditioning Research, 33 (2019) 2019-2052. [DOI] [PubMed]

[4] H. Chaabene, M. Lesinski, D. Behm, U. Granacher, Performance - and health-related benefits of youth resistance training, Sports Orthopaedics and Traumatology, 36(3) (2020) 231-240. [DOI]

[5] M. Lesinski, O. Prieske, \& U. Granacher, Effects and dose-response relationships of resistance training on physical performance in youth athletes: a systematic review and meta-analysis, British Journal of Sports Medicine, 50(13) (2016) 781-795. [DOI] [PubMed]

[6] A, Winett, Richard \& Carpinelli, N. Ralph, Potential Health-Related Benefits of Resistance Training, Preventive Medicine, 33(5) (2001) 503513. [DOI] [PubMed]

[7] Majid Mufaqam Syed-Abdul, Benefits of Resistance Training in Older Adults, Current Aging Science, 14(1) (2021) 5-9. [DOI] [PubMed]

[8] Kawamori, Naoki \& Newton, Robert \& Nosaka, Ken, Effects of weighted sled towing on ground reaction force during the acceleration phase of sprint running, Journal of Sports Sciences, 32(12) (2014) 1139-45. [DOI] [PubMed]

[9] Rosario, Martin, Neuromuscular timing modification in responses to increased speed and proportional resistance while pushing a sled in young adults, European Journal of Human Movement, 44 (2020). [DOI]

[10] G.S. Nunes, T. Pizzari, R. Neate, C.J. Barton, \& A. Semciw, Gluteal muscle activity during running in asymptomatic people, Gait \& posture, 80 (2020) 268-273. [DOI] [PubMed]

[11] J.D. Willson, T.W. Kernozek, R.L. Arndt, D.A. Reznichek, \& J. Scott Straker, Gluteal muscle activation during running in females with and without patellofemoral pain syndrome. Clinical biomechanics (Bristol, Avon), 26(7), (2011) 735740. [DOI] [PubMed]

[12] Rosario, Martin G, \& Mathis, Monica, Lower limb muscle activation and kinematics modifications of young healthy adults while pushing a variable resistance sled, Journal of Human Sport and Exercise, 16(4) (2020a). [DOI]

[13] Y.J. Lee, B. Chen, \& A.S. Aruin, Older adults utilize less efficient postural control when performing pushing task, Journal of electromyography and kinesiology : official journal of the International Society of Electrophysiological Kinesiology, 25(6) (2015) 966-972. [DOI] [PubMed]

[14] M. Powers, Christopher, The influence of abnormal hip mechanics on knee injury: a biomechanical perspective, The Journal of Orthopaedic and Sports Physical Therapy, 40(2) (2010) 42-51. [DOI] [PubMed]

[15] W.K. Neto, E.G. Soares, T.L. Vieira, R. Aguiar, T.A. Chola, V.L. Sampaio, \& E.F. Gama, Gluteus Maximus Activation during Common Strength and Hypertrophy Exercises: A Systematic Review, Journal of Sports Science \& Medicine, 19(1) (2020) 195-203. [PubMed]

\section{Funding}

No funding was received to carry out this study

\section{Authors Contribution}

All the authors equally contributed to this work, read and approved the final version of the manuscript.

\section{Ethics Approval}

IRB approval TWU protocol \# 20092

\section{Informed Consent}

Written consent obtained from the participants

\section{Conflict of interest}

The Authors have no conflicts of interest to declare that they are relevant to the content of this article.

\section{Does this article screened for similarity? Yes}

\section{About The License}

(c) The Author(s) 2021. The text of this article is open access and licensed under a Creative Commons Attribution 4.0 International License 